\title{
Evaluation of the antioxidant activity of Coreopsis tinctoria Nuff. and optimisation of isolation by response surface methodology
}

\author{
SHUMIN LAN ${ }^{1}$ \\ JINGMING LIN $^{2}$ \\ NI ZHENG ${ }^{1 *}$ \\ ${ }^{1}$ Department of Pharmacy, Guangdong \\ No. 2 Provincial People's Hospital \\ Guangzhou 510317, PR China \\ ${ }^{2}$ Department of Pharmacy, Zhujiang \\ Hospital, Southern Medical University \\ Guangzhou 510282, PR China
}

\begin{abstract}
The aim of the present study was to determine the antioxidant activity of Coreopsis tinctoria flowering tops (CTFT). Studies were conducted to obtain suitable extraction conditions for chlorogenic acid, quercetin, luteolin, apigenin and kaempferol, which were identified and quantified by HPLC. Response surface methodology was employed to optimise the ultrasound-assisted extraction conditions including extraction time, ethanol concentration and liquid-solid ratio. The antioxidant activity of the extracts was analysed using various antioxidant models, such as DPPH, ABTS and hydroxyl radical scavenging assay. CTFT extracted for $15.0 \mathrm{~min}$ with ethanol at a concentration of $60.4 \%$ and with liquidsolid ratio 27.5:1 possessed a considerable amounts of total flavonoids and polyphenols (18.9\%). This extract showed higher scavenging activity of ABTS and hydroxyl radical activity than rutin, however not in the DPPH test. We may assume that CTFT possess antioxidant and free radical scavenging potentials.
\end{abstract}

Keywords: Coreopsis tinctoria (Asteraceae), flavonoids, polyphenols, antioxidant activity, response surface methodology, HPLC

Coreopsis tinctoria Nutt. is a plant native to North America but spread worldwide. North American Indians have used it to treat several disorders. They traditionally used root tea against diarrhea and as an emetic and dried tops for blood, internal pains and bleeding (1). In Portugal, C. tinctoria flowering tops infusion has been traditionally used to reduce hyperglycaemia in diabetic patients. Its use is also documented in traditional Chinese medicine where a decoction of the whole plant is used for diarrhoea (2) and diabetes (3). The aim of this study was to elucidate the chemical composition of Coreopsis tinctoria flowering tops by HPLC after optimisation of ultrasound-assisted extraction by response surface methodology (RSM). In addition, evaluation of the antioxidant capacity of ethanol extracts by 1,1-diphenyl-2-picrylhydrazyl (DPPH), 2,2-azino-bis (3-ethyl-benzthiazoline-6sulphonic acid) diammonium salt (ABTS) and hydroxyl radical assay were undertaken.

\footnotetext{
*Correspondence; e-mail address: zhengni8787@163.com
} 


\section{EXPERIMENTAL}

\section{Plant materials and apparatus}

Coreopsis tinctoria Nutt. (Asteraceae) flowering tops were acquired from Guangzhou crude medicine companies and identified at the College of Life Science and Biopharmacology, Guangdong Pharmaceutical University, Guangdong (China). To avoid degradation, the air-dried plant material was ground just before extraction.

\section{Chemicals and reagents}

$\mathrm{DPPH}, \mathrm{ABTS}$ and crocus (used in the hydroxyl radical scavenging assay) were obtained from Sigma-Aldrich, Germany. Ascorbic acid was purchased from Sigma Chemical Co. (USA). Water was purified using a Milli-Q water purification system (Milipore, USA). Standards of chlorogenic acid, quercetin, luteolin, apigenin, kaempferol and rutin were purchased from the National Institute for the Control of Pharmaceutical and Biological Products (China). All other chemicals and solvents used for extraction were analytical grade and were obtained from Tianjin Reagent Company (China).

\section{Extraction of Coreopsis tinctoria flowering tops}

Coreopsis tinctoria flowering tops were crushed in a $0.7 \mathrm{~mm}$ screen. To dried powder (5.0 g), the solvent was added (ethanol of 40, 60 or $80 \%$ ) in a defined liquid-solid ratio (20:1, 30:1, 40:1) and extracted with in ultrasonic bath for defined time intervals (15, 30 and 45 $\min )$ at $50 \mathrm{kHz}$. The filtrate was collected and the residue was extracted two times according to the protocol given above. The extract was then concentrated to no ethanol by rotary vaporisation at $60^{\circ} \mathrm{C}$ under reduced pressure and residue was constant to a certain volume by methanol. Then, the filtered solution was stored at $4{ }^{\circ} \mathrm{C}$ for further analysis.

\section{HPLC analysis}

Concentrations of the main flavonoid and polyphenol compounds were determined by calculating the area under the HPLC peak and presented as the mean of two measurements. The extracts were quantified using an Agilent 1100 HPLC system equipped with ChemStation software (Agilent Technologies, USA), a quaternary pump, an online vacuum degasser, an autosampler, a thermostated column compartment and a diode array detector. Separation was achieved on a Hypersil ODS C18 column $(4.6 \mathrm{~mm} \times 250 \mathrm{~mm}, 5 \mu \mathrm{m})$ from Dalian Elite Analytical Instruments Co. Ltd. (China). Column temperature was set at $25^{\circ} \mathrm{C}$ and the detection wavelength was $360 \mathrm{~nm}$. The mobile phase consisted of methanol and $0.15 \%$ phosphoric acid solution $(45: 55, V / V)$. Flow rate was $1.0 \mathrm{~mL} \mathrm{~min}{ }^{-1}$. The injection volume was $10 \mu \mathrm{L}$. All the main flavonoid and polyphenolic compounds were identified by matching their retention times against those of standard compounds.

\section{Box-Behnken design for response surface methodology}

To obtain suitable extraction conditions for Coreopsis tinctoria, Box-Behnken designs (BBDs, Design Expert software, Trial Version 8.0.4, Stat-Ease Inc., Minneapolis, MN, USA) 
were applied to the experimental design, data analysis and model building. Box-Behnken designs for multivariate optimization are a class of rotatable or nearly rotatable secondorder designs based on the three-level incomplete factorial design (4). $R^{2}$ (adjusted determination coefficient) is the measure for determining the goodness-of-fit of the regression equation (5).

Based on the preliminary tests, a total of 17 runs from BBDs were employed to optimise the main extraction conditions, including the extraction time $\left(X_{1}\right)$, ethanol concentration $\left(X_{2}\right)$ and liquid-solid ratio $\left(X_{3}\right)$, as shown in Table I.

Table I. Levels of extraction in designed experiments

\begin{tabular}{cccc}
\hline Parameter/level & $\begin{array}{c}\text { Extraction } \\
\text { time }\left(\min , X_{1}\right)\end{array}$ & $\begin{array}{c}\text { Ethanol } \\
\text { concentration }\left(\%, X_{2}\right)\end{array}$ & $\begin{array}{c}\text { Liquid-to-solid } \\
\text { ratio }\left(X_{3}\right)\end{array}$ \\
\hline-1 & 15 & 40 & 20 \\
0 & 30 & 60 & 30 \\
1 & 45 & 80 & 40 \\
\hline
\end{tabular}

\section{Antioxidant activity}

Scavenging capacity on DPPH radical. - Free radical scavenging activity of each extract and rutin was determined based on the extract's ability to react with the stable DPPH free radical. In brief, $2.5 \mathrm{~mL}$ of the extract was added to $2.5 \mathrm{~mL}$ of DPPH $\left(250 \mu \mathrm{g} \mathrm{mL}^{-1}\right.$, dissolved in $95 \%$ ethanol). The mixture was vigorously shaken and maintained at room temperature for $30 \mathrm{~min}$ in the dark. Absorbance was measured using a spectrophotometer (Lambda 35 UV-visible, Perkin Elmer, USA) at $517 \mathrm{~nm}$ against a blank.

ABTS radical scavenging assay. - The antioxidant activity was determined using ABTS radical cation measurements $(6-8)$, with some modifications. ABTS radical cations were produced by reacting $7 \mathrm{mmol} \mathrm{L}^{-1}$ ABTS solution with $2.45 \mathrm{mmol} \mathrm{L}^{-1}$ potassium persulfate. The mixture was stored in the dark, at room temperature, for $16 \mathrm{~h}$. Upon use, the ABTS solution was diluted with ethanol to an absorbance value of $0.70 \pm 0.02$ at $734 \mathrm{~nm} .0 .3 \mathrm{~mL}$ of each extract sample of varying concentration was added to $3.0 \mathrm{~mL}$ of ABTS solution and mixed vigorously. The reaction mixture was allowed to stand at room temperature for 6 min and the absorbance at $734 \mathrm{~nm}$ was immediately measured. The percentual ABTS scavenging effect was calculated.

Hydroxyl radical scavenging assay. - The hydroxyl radical scavenging activity of each extract was measured according to the method described by Wang et al. (9-11) with some modifications. Extract samples were incubated with $2.0 \mathrm{mmol} \mathrm{L}^{-1}$ EDTA-Fe $(0.5 \mathrm{~mL}), 3 \%$ $\mathrm{H}_{2} \mathrm{O}_{2}(1.0 \mathrm{~mL})$ and $360 \mu \mathrm{g} \mathrm{mL} \mathrm{m}^{-1}$ crocus in $4.5 \mathrm{~mL}$ sodium phosphate buffer $\left(150.0 \mathrm{mmol} \mathrm{L}^{-1}\right.$, $\mathrm{pH}$ 7.4) for $30 \mathrm{~min}$ at $37^{\circ} \mathrm{C}$. Hydroxyl radical was detected by monitoring absorbance at 520 $\mathrm{nm}$. The percentual hydroxyl radical scavenging effect was calculated. In the control, the sample was substituted by distilled water and sodium phosphate buffer was replaced with $\mathrm{H}_{2} \mathrm{O}_{2}$. 
a)

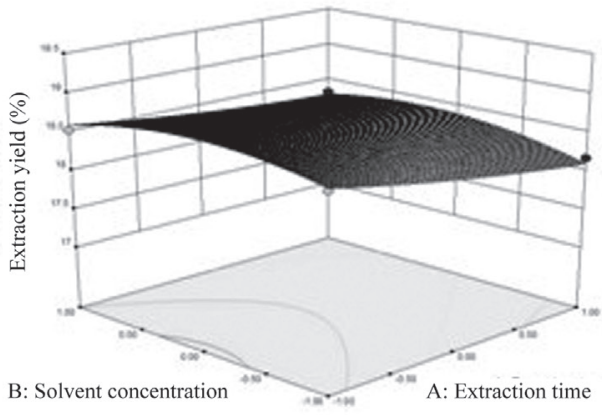

c)
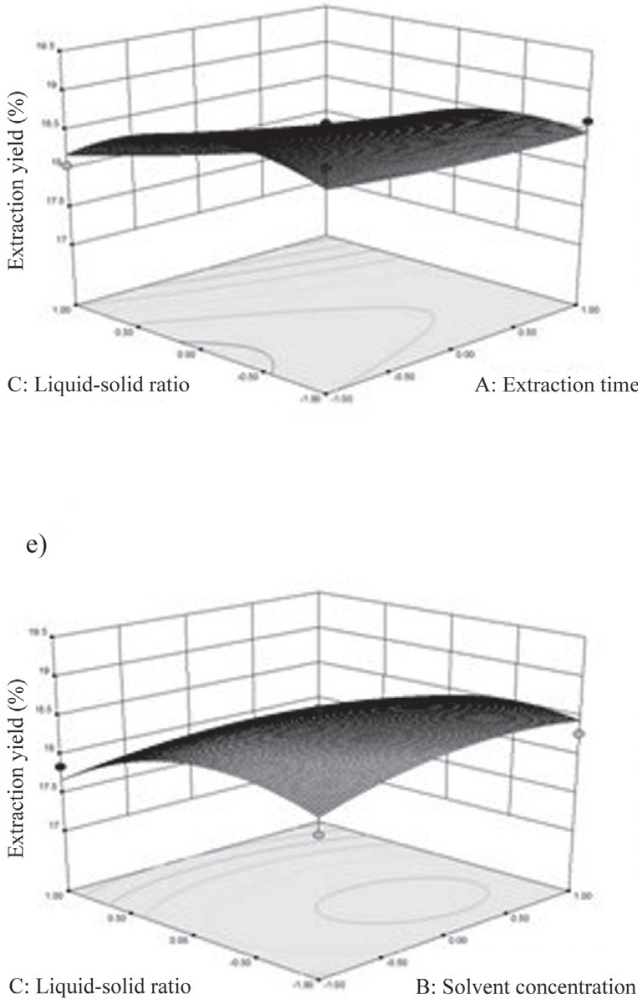

b)

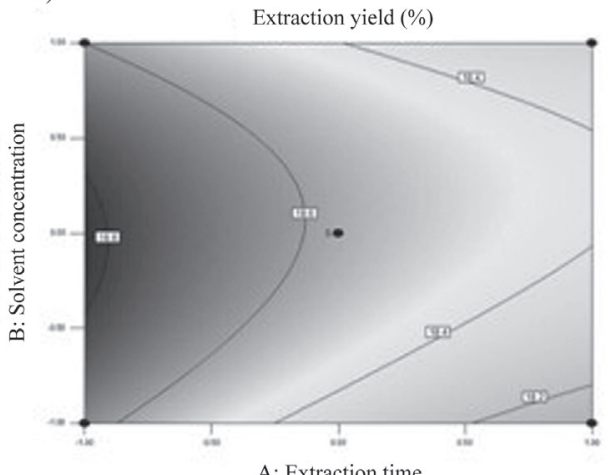

A: Extraction time

d)

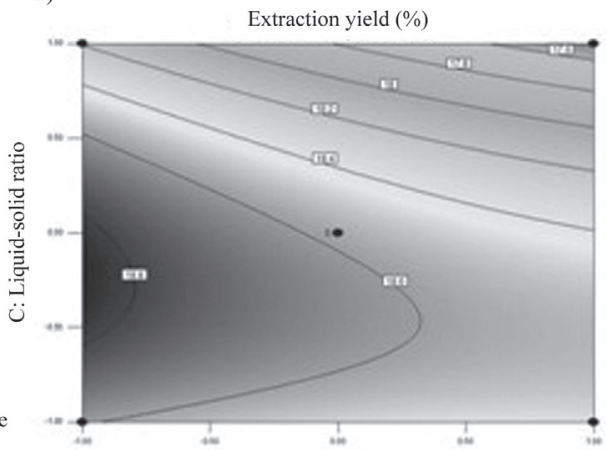

A: Extraction time

f)

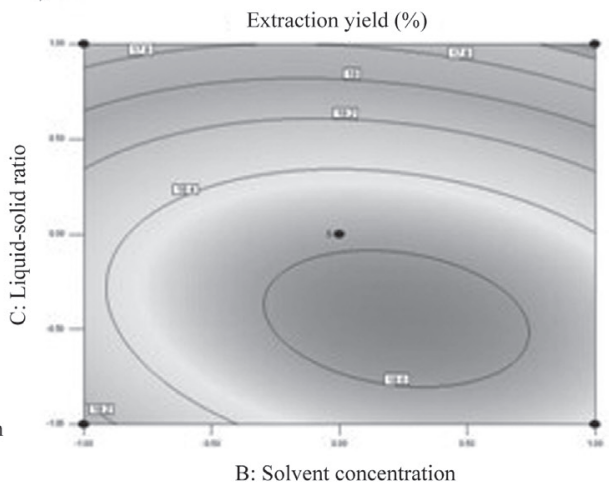

Fig. 1. Response surface plots and contour plots of extraction time (minutes, min), ethanol concentration $(\%, V / V)$ and solid-to-liquid ratio showing their effect on extraction yield. 


\section{Statistical analysis}

All experimental data (in tables and figures) represent mean values \pm standard deviation $(n=3)$. Statistical significance was set at a value of 0.05 . The response obtained from each set within experimental design was subjected to multiple non-linear regressions using the Design Expert software, Trial Version 8.0.4 (Stat-Ease, Minneapolis, USA). The quality of the fit of the polynomial model equation was checked using the $F$-test and $p$-value.

\section{RESULTS AND DISCUSSION}

\section{Optimiszation of response surface methodology (RSM)}

The results indicated that the RSM model used to fit the response variable was significant $(p=0.0135)$ and adequate to represent the relationship between the response and the independent variables $(12,13)$. The model demonstrated a very high $F$-value $(F=6.0442)$. The $R^{2}$ value of the RSM model was 0.986 , which indicates that only $1.40 \%$ of the total variations were not covered by the model. Meanwhile, relative standard deviation $(R S D=0.8 \%)$ demonstrated good precision of the experiments performed (14).

Table II shows that the extraction yield was mostly affected by the liquid-solid ratio $\left(X_{3}, p=0.0023\right)$, followed by extraction time $\left(X_{1}, p=0.0275\right)$. Ethanol concentration $\left(X_{2}\right)$ was not found to affect the extraction yield significantly. It was evident that $X_{3}{ }^{2}$ was significant at the level of $p<0.01$. The predicted response $Y$ for the extraction yield could be expressed by the following second-order polynomial equation:

$$
\begin{gathered}
Y=18.57-0.21 X_{1}+0.037 X_{2}-0.35 X_{3}+0.060 X_{1} X_{2}-0.14 X_{1} X_{3}-0.12 X_{2} X_{3}+0.047 X_{1}^{2}- \\
-0.02 X_{2}^{2}-0.43 X_{3}^{2}
\end{gathered}
$$

Regression equation was graphically represented by the 3D response surface and 2D contour plots (15). From the 3D response surface curves and contour plots shown in Fig. 2, the effect of the independent variables and their mutual interaction on the extraction yield can be seen. Figs. 2a and b represent the response surface and contour plots showing the effect of the extraction time and ethanol concentration at a fixed liquid-to-solid ratio. As shown in the figure, the extraction yield decreased by increasing the extraction time. Any decrease in the extraction time had only a slight effect on extraction yield. Figs. $2 \mathrm{c}$ and $\mathrm{d}$ depict the interaction of the extraction time and the liquid-to-solid ratio on the extraction yield at fixed ethanol concentration. By increasing the liquid-solid ratio, the yield reached a maximum, and beyond this point, no increase in the liquid-to-solid ratio improved the yield. Figs. 2e and $f$ describe the effect of the ethanol concentration and liquid-to-solid ratio on the yield at a fixed extraction time. By increasing the ethanol concentration, the yield reached a maximum, and beyond this point, no increase in the ethanol concentration improved the yield.

One aim of this study was to indentify the conditions that produced the maximum extraction yield in Coreopsis tinctoria. According to the results of designed experiments, the software predicted the optimum extraction time, ethanol concentration and liquid-to-solid ratio to be $15.0 \mathrm{~min}, 60.4 \%$ and $27.5: 1$, respectively. Under these conditions, the extraction yield of all five main flavonoid and polyphenol compounds (chlorogenic acid, quercetin, 

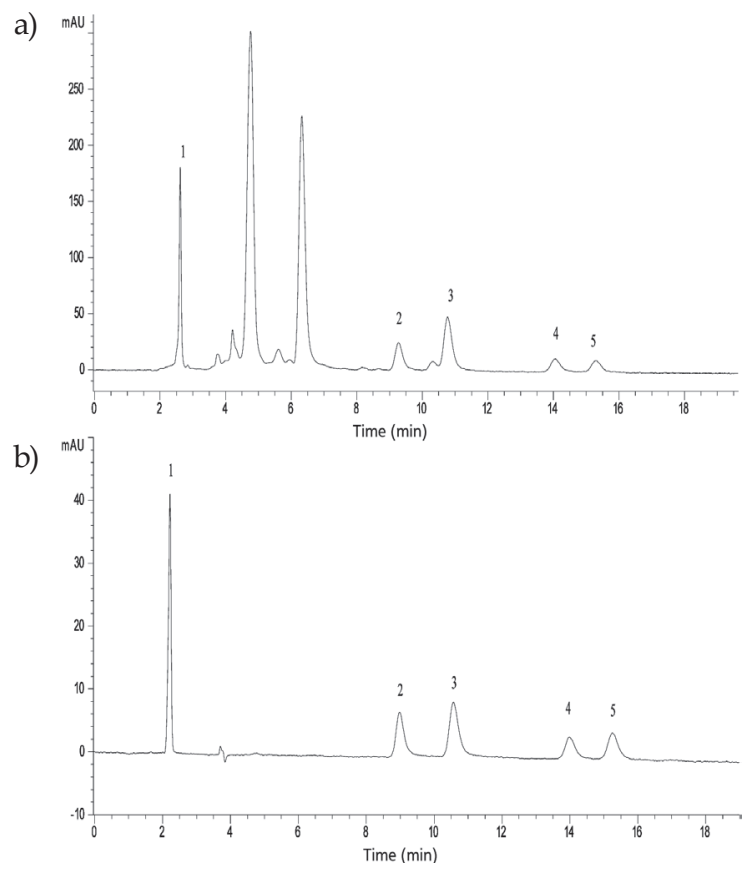

Fig. 2. HPLC chromatogram at $360 \mathrm{~nm}$ for: a) standard solution of five flavonoids and polyphenols, b) Coreopsis tinctoria flowering top extract. Keys to the peaks: 1 - chlorogenic acid, 2 - quercetin, 3 - luteolin, 4 - apigenin and 5 - kaempferol.

luteolin, apigenin and kaempferol) predicted by the software was $18.85 \%$. To test the validity of the response surface analysis, the extraction was carried out under the proposed conditions and the extraction yield was determined to be $18.87 \pm 0.52 \%(n=3)$. The high correlation between these results confirmed that the response model was adequate to predict optimal operating conditions.

\section{Identification and quantification of extract constituents}

Five compounds were identified, namely, chlorogenic acid, quercetin, luteolin, apigenin and kaempferol, on the basis of their retention times compared to the standards. To confirm linearity, standard solutions in the concentration range were prepared and injected into the HPLC system (Table III). The HPLC profiles of flavonoids and phenolic acids extracted from Coreopsis tinctoria are shown in Fig. 1.

\section{Antioxidant activity}

Scavenging of DPPH radical. - Our results indicate that CTFT extract and rutin were effective in reducing the stable radical DPPH to the yellow-coloured diphenylpicrylhydrazine (Fig. 3a). CTFT active components had significant scavenging effects with the increasing concentration of active components (range of $0.05-1.6 \mu \mathrm{mol} \mathrm{L}^{-1}$ ). However, the scaveng- 
S. Lan et al.: Evaluation of the antioxidant activity of Coreopsis tinctoria Nuff. and optimisation of isolation by response surface methodology, Acta Pharm. 64 (2014) 369-378.

Table II. Analysis of variance (ANOVA) for experimental results

\begin{tabular}{|c|c|c|c|c|c|}
\hline Factor & $\begin{array}{l}\text { Sum of squares } \\
\text { for error }\end{array}$ & $\begin{array}{l}\text { Mean square } \\
\text { error }\end{array}$ & $\mathrm{df}$ & $F$-value & $p$-value \\
\hline Model & 2.4879 & 0.2764 & 9 & 6.0442 & 0.0135 \\
\hline$X_{1}$ & 0.3519 & 0.3519 & 1 & 7.6953 & 0.0275 \\
\hline$X_{2}$ & 0.0110 & 0.0110 & 1 & 0.2411 & 0.6384 \\
\hline$X_{3}$ & 0.9877 & 0.9877 & 1 & 21.5955 & 0.0023 \\
\hline$X_{1} X_{2}$ & 0.0143 & 0.0143 & 1 & 0.3122 & 0.5937 \\
\hline$X_{1} X_{3}$ & 0.0792 & 0.0792 & 1 & 1.7325 & 0.2295 \\
\hline$X_{2} X_{3}$ & 0.0595 & 0.0595 & 1 & 1.3017 & 0.2914 \\
\hline$X_{1}^{2}$ & 0.0092 & 0.0092 & 1 & 0.2008 & 0.6676 \\
\hline$X_{2}{ }^{2}$ & 0.1762 & 0.1762 & 1 & 3.8518 & 0.0905 \\
\hline$X_{3}^{2}$ & 0.7661 & 0.7660 & 1 & 16.7497 & 0.0046 \\
\hline Lack of fit & 0.3184 & 0.1061 & 3 & 236.9869 & $<0.0001$ \\
\hline Error & 0.0018 & 0.0004 & 4 & & \\
\hline Total & 2.8081 & & 16 & & \\
\hline
\end{tabular}

df - degrees of freedom

Table III. Linear regression data

Standard solution Correlation coefficient $(R) \quad$ Molar concentration $\left(\mu \mathrm{mol} \mathrm{L}^{-1}\right)$

\begin{tabular}{lll}
\hline Chlorogenic acid & 0.9992 & $0.189-7.795$ \\
Quercetin & 0.9991 & $0.278-10.788$ \\
Luteolin & 0.9900 & $0.238-6.407$ \\
Apigenin & 0.9997 & $0.241-6.520$ \\
Kaempferol & 0.9993 & $0.307-7.602$ \\
\hline
\end{tabular}

ing effect of CTFT was significantly lower than that of rutin. At $200 \mu \mathrm{g} \mathrm{mL}{ }^{-1}$, active components in CTFT and rutin exhibited 68.7 and $89.5 \%$ inhibition, respectively, and the $E C_{50}$ values were 93.29 and $42.43 \mu \mathrm{g} \mathrm{mL}^{-1}$ for active components in CTFT and rutin, respectively (Table III). Both CTFT and rutin showed antioxidant activities in a dose dependent manner in DPPH radical scavenging.

Savenging ABTS radical. - Scavenging power correlated well with increasing concentrations of active components. Aliquots of CTFT or rutin $\left(100 \mu \mathrm{g} \mathrm{mL} \mathrm{mL}^{-1}\right)$ exhibited 73.7 and 
a)

Rutin

CTFT

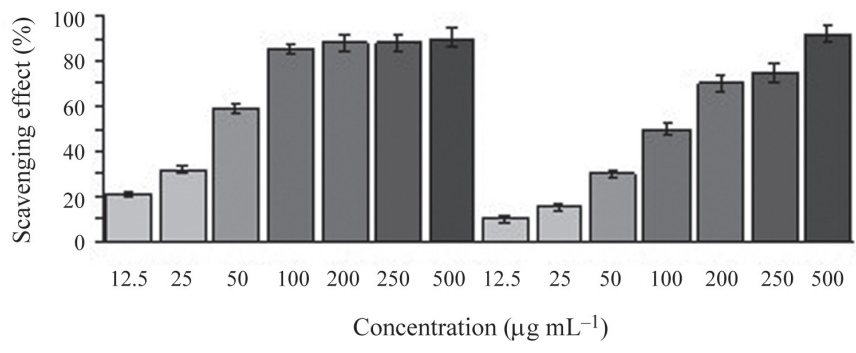

b)

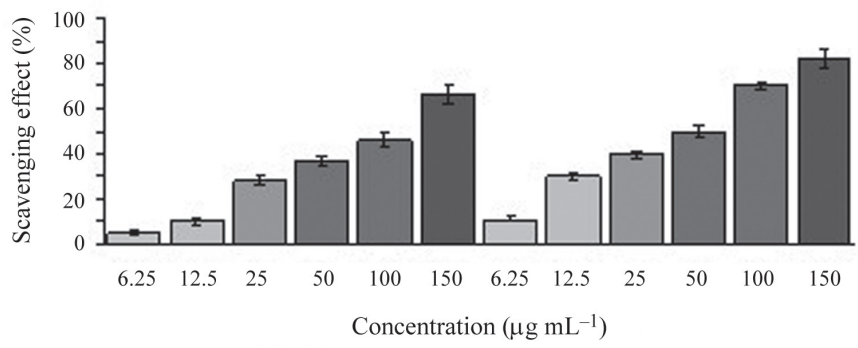

c)

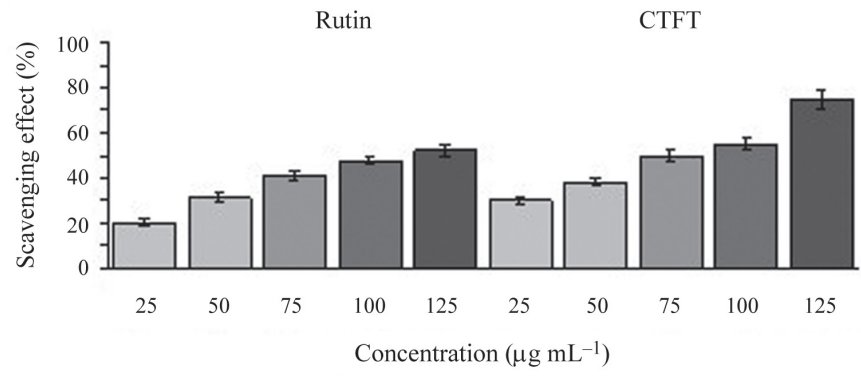

Fig. 3. Radical-scavenging activity of active components from Coreopsis tinctoria flowering tops (CTFT) and rutin. Radical-scavenging activities were assessed by measuring: a) DPPH, b) ABTS and c) hydroxyl scavenging activity (mean $\pm \mathrm{SD}, n=3$ ).

$49.1 \%$ inhibition, respectively. The $E C_{50}$ value of CTFT for ABTS radical scavenging activity was $46.29 \mu \mathrm{g} \mathrm{mL}^{-1}$, whereas the $E C_{50}$ value of rutin was found to be $132.47 \mu \mathrm{g} \mathrm{mL}^{-1}$.

Scavenging effects on hydroxyl radicals. - All results showed antioxidant activity in a dose dependent manner. The $E C_{50}$ values of CTFT and rutin were 66.17 and $122.25 \mu \mathrm{g} \mathrm{mL}^{-1}$, respectively (Table III). The ability of CTFT extracts to quench hydroxyl radicals seems to be directly related to the prevention of propagation of lipid peroxidation. Since CTFT seems to be a good scavenger of active oxygen species, it will reduce the rate of hydroxyl radicals' chain reaction. 
S. Lan et al.: Evaluation of the antioxidant activity of Coreopsis tinctoria Nuff. and optimisation of isolation by response surface methodology, Acta Pharm. 64 (2014) 369-378.

Table IV. Effect of active components from Coreopsis tinctoria on different radical scavenging activities

\begin{tabular}{lccc}
\hline \multirow{3}{*}{ Samples } & \multicolumn{3}{c}{$E C_{50}\left(\mu \mathrm{g} \mathrm{mL}^{-1}\right)^{\mathrm{a}}$} \\
\cline { 2 - 4 } & DPPH & \multicolumn{1}{c}{ ABTS } & Hydroxyl \\
\hline CTFT & $93.29 \pm 1.56$ & $46.29 \pm 1.31$ & $66.17 \pm 1.15$ \\
Rutin & $42.43 \pm 2.13$ & $132.47 \pm 1.85$ & $122.25 \pm 1.39$ \\
\hline
\end{tabular}

$E C_{50}-$ measure of radical scavenging activity being the concentration required to inhibit $50 \%$ free radical activity.

${ }^{a}$ Mean \pm SD values $(n=3)$.

\section{CONCLUSIONS}

In the present study, we demonstrated that CTFT possesses considerable amounts of flavonoids and polyphenols (18.9\%). The data obtained clearly indicate that the extract possesses potent ABTS and hydroxyl radical scavenging activity. CTFT was also able to scavenge DPPH. These antioxidant activities could have contributed, at least partly, to the therapeutic benefits of certain traditional claims for Coreopsis tinctoria flowering tops. In view of the potential use of Coreopsis tinctoria flowering tops in the functional food industry, its therapeutic benefits and bioactive compounds warrant further investigation.

\section{REFERENCES}

1. T. Dias, M. R. Bronze, P. J. Houghton, H. M. Filipe and A. Paulo, The flavonoid-rich fraction of Coreopsis tinctoria promotes glucose tolerance regain through pancreatic function recovery in streptozotocin-induced glucose-intolerant rats, J. Ethnopharmacol. 132 (2010) 483-490; DOI: 10.1016/j. jep.2010.08.048.

2. Y. Zhang, S. Shi, M. Zhao, X. Chai and P. F. Tu, Coreosides A-D, $C_{14}$-polyacetylene glycosides from the capitula of Coreopsis tinctoria and its anti-inflammatory activity against COX-2, Fitoterapia 87 (2013) 93-97; DOI: 10.1016/j.fitote.2013.03.024.

3. T. Dias, B. Liu, P. Jones, P. J. Houghton, H. M. Filipe and A. Paulo, Cytoprotective effect of Coreopsis tinctoria extracts and flavonoids on $\mathrm{tBHP}$ and cytokine-induced cell injury in pancreatic MIN6 cells, J. Ethnopharmacol. 139 (2012) 485-492; DOI: 10.1016/j.jep.2011.11.038.

4. G. L. Li, X. L. Zhang, J. M. You, C. H Song, Z. W. Sun, L. Xia and Y. R. Suo, Highly sensitive and selective pre-column derivatization high-performance liquid chromatography approach for rapid determination of triterpenes oleanolic and ursolic acids and application to Swertia species: optimization of triterpenic acids extraction and pre-column derivatization using response surface methodology, Anal. Chim. Acta 688 (2011) 208-218; DOI: 10.1016/j.aca.2011.01.010.

5. Y. Li, J. G. Jiang, W. F. Li, C. Jian, D. Y. Wang and L. Zhu, Optimum extraction process of polyphenols from the bark of Phyllanthus emblica L. based on the response surface methodology, J. Sep. Sci. 32 (2009) 1437-1444; DOI: 10.1002/jssc.200800744.

6. A. X. Luo, X. J. He, S. D. Zhou, Y. J. Fan, A. S. Luo and Z. Chun, Purification, composition analysis and antioxidant activity of the polysaccharides from Dendrobium nobile Lindl, Carbohyd. Polym. 79 (2010) 1014-1019; DOI: 10.1016/j.carbpol.2009.10.033. 
7. P. C. Wootton-Beard, A. Moran and L. Ryan, Stability of the total antioxidant capacity and total polyphenol content of 23 commercially available vegetable juices before and after in vitro digestion measured by FRAP, DPPH, ABTS and Folin-Ciocalteu methods, Food Res. Int. 44 (2011) 217224; DOI: 10.1016/j.foodres.2010.10.033.

8. Y. J. Fan, X. J. He, S. D. Zhou, A. X. Luo, T. He and Z. Chun, Composition analysis and antioxidant activity of polysaccharide from Dendrobium denneanum, Int. J. Biol. Macromol. 45 (2009) 169-173; DOI: 10.1016/j.ijbiomac.2009.04.019.

9. İ. Gülçin, Antioxidant and antiradical activities of L-carnitine, Life Sci. 78 (2006) 803-811; DOI: 10.1016/j.lfs.2005.05.103.

10. M. Marzouk, A. M. Soliman and T. Y. Omar, Hypoglycemic and antioxidative effects of fenugreek and termis seeds powder in streptozotocin-diabetic rats, Eur. Rev. Med. Pharmacol. Sci. 17 (2013) $559-565$.

11. J. Wang, Q. B. Zhang, Z. S. Zhang and Z. E. Li, Antioxidant activity of sulfated polysaccharide fractions extracted from Laminaria japonica, Int. J. Biol. Macromol. 42 (2008) 127-132; DOI: 10.1016/j. ijbiomac.2007.10.003.

12. T. S. Ballard, P. Mallikarjunan, K. Q. Zhou and S. F. O'Keefe, Optimizing the extraction of phenolic antioxidants from peanut skins using response surface methodology, J. Agric. Food Chem. 57 (2009) 3064-3072; DOI: 10.1021/jf8030925.

13. M. K. Pierozan, R. J. da Costa, O. A. Antunes, E. G. Oestreicher, J. V. Oliveira, R. L. Cansian, H. Treichel and de D. Oliveira, Optimization of extraction of lipase from wheat seeds (Triticum aestivum) by response surface methodology, J. Agric. Food Chem. 57 (2009) 9716-9721; DOI: 10.1021/ jf901816x.

14. P. Thana, S. Machmudah, M. Goto, M. Sasaki, P. Pavasant and A. Shotipruk, Response surface methodology to supercritical carbon dioxide extraction of astaxanthin from Haematococcus pluvialis, Bioresour. Technol. 99 (2008) 3110-3115; DOI: 10.1016/j.biortech.

15. W. Li, Z. Wang, Y.-S. Sun, L. Chen, L.-K. Han and Y.-N. Zheng, Application of response surface methodology to optimise ultrasonic-assisted extraction of four chromones in Radix Saposhnikoviae, Phytochem. Anal. 22 (2011) 313-321; DOI: 10.1002/pca.1282. 